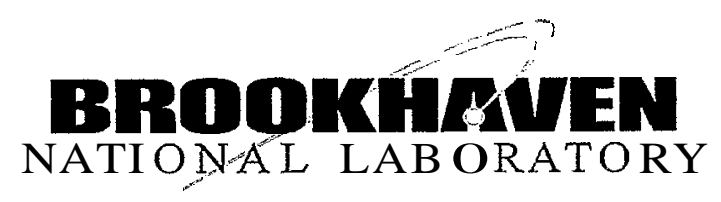

BNL-73470-2005-CP

\title{
Comparison of Off-line IR Bump and Action-Angle Kick Minimization
}

\author{
Y. Luo, F. Pilat, V. Ptitsyn, D. Trbojevic, J. Wei \\ Presented at the Particle Accelerator Conference(PAC'05) \\ Knoxville, Tennessee \\ May 16-20, 2005
}

Collider-Accelerator Department

Brookhaven National Laboratory

P.O. Box 5000

Upton, NY 11973-5000

WWw.bnl.gov

Managed by

Brookhaven Science Associates, LLC

for the United States Department of Energy under

Contract No. DE-AC02-98CH10886

This is a preprint of a paper intended for publication in a journal or proceedings. Since changes may be made before publication, this preprint is made availablewith the understanding that it will not be cited or reproduced without the permission of the author. 


\section{DISCLAIMER}

This report was prepared as an account of work sponsored by an agency of the United States Government. Neither the United States Government nor any agency thereof, nor any of their employees, nor any of their contractors, subcontractors, or their employees, makes any warranty, express or implied, or assumes any legal liability or responsibility for the accuracy, completeness, or any third party's use or the results of such use of any information, apparatus, product, or process disclosed, or represents that its use would not infringe privately owned rights. Reference herein to any specific commercial product, process, or service by trade name, trademark, manufacturer, or otherwise, does not necessarily constitute or imply its endorsement, recommendation, or favoring by the United States Government or any agency thereof or its contractors or subcontractors. The views and opinions of authors expressed herein do not necessarily state or reflect those of the United States Government or any agency thereof.

FOR UNCLASSIFIED, UNLIMITED STI PRODUCTS

Available electronically at:

OSTI:

\section{http://www.osti.gov/bridge}

Available for a processing fee to U.S. Department of Energy and its contractors, in paper from:

U.S. Department of Energy

Office of Scientific and Technical Information

P.O. Box 62

Oak Ridge, TN 37831

Phone: (865) 576-8401

Facsimile: (865) 576-5728

E-mail: reports@adonis.osti.gov

National Technical Information Service (NTIS):

Available for sale to the public from:

U.S. Department of Commerce

National Technical Information Service

5285 Port Royal Road

Springfield, VA 22131

Phone: (800) 553-6847

Facsimile: (703) 605-6900

Online ordering: http://www.ntis.gov/ordering.htm

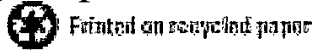




\title{
Comparison of Off-line IR Bump and Action-Angle Kick Minimization*
}

\author{
Y. Luo, F. Pilat, V. Ptitsyn, D. Trbojevic, J. Wei \\ Brookhaven National Laboratory, Upton, NY 11973, USA
}

\begin{abstract}
The interaction region bump (IR bump) nonlinear correction method has been used for the sextupole and octupole field error on-line corrections in the Relativistic Heavy Ion Collider (RHIC). Some differences were found for the sextupoleand octupole corrector strengths between the on-line IR bump correction and the predictions from the action-anglekick minimization. In this article, we compare the corrector strengths from these two methods based on the RHIC Blue ring lattice with the $\mathbb{R}$ nonlinear modeling. The comparison confirms the differences between resulting corrector strengths. And the reason for the differences is found and discussed.
\end{abstract}

\section{ACTION-ANGLE KICK MINIMIZATION}

To minimize the action change for each order of IR nonlinear field error, it is equivalentto minimize the following quantities simultaneously[1],

$$
\oint_{\mathbf{L}} d s C_{z} c_{n}+(-1)^{n+1} \oint_{R} d s C_{z} c_{n}
$$

where $L$ and $\mathrm{R}$ mean the left and right sides of the interaction region, $z$ stands for $\boldsymbol{x}$ or $\boldsymbol{y}$ plane, $c_{n}$ stands for the normal or skew field errors $b_{n}$ or $a_{n}, n$ is the field error order. $C_{z}$ is the weight factor, which is different for different order of errors and for different $x$ or y plane. The integrations or summations are taken across the interaction region. The correction weight factors are given according to the multiples as

$$
\begin{aligned}
C_{x} & = \begin{cases}\beta_{x}^{(n+1) / 2} & \text { for } b_{n} \\
\beta_{x}^{n / 2} \beta_{y}^{1 / 2} & \text { for } a_{n}\end{cases} \\
C_{y} & = \begin{cases}\beta_{y}^{(n+1) / 2} & \text { for odd } b_{n} \text { and even } a_{n} \\
\beta_{x}^{1 / 2} \beta_{y}^{n / 2} & \text { for even } b_{n} \text { and odd } a,\end{cases}
\end{aligned}
$$

For each order, there are two quantities to be minimized, one in the horizontalplane, one in the vertical plane. Therefor, at least two nonlinear correctors are needed for each order correction. A natural choice is to place one corrector in either side of the interaction region. In order to facilitate the following discussion, Table 1 lists the weightfactors for different field error $b$, and $a$.

The action-angle kick minimization assumes that the phase advances in the horizontal and vertical planes across the interaction point are closeto $\pi$. And it ignores the phase advance in the triplet because of the small $\beta^{*}$. It assumes the beam is round beam, therefore, only the leading resonances in horizontal and vertical planes are corrected.

\footnotetext{
*Work supported by U.S. DOE under contract No DE-AC0298CH10886
}

Table 1: Weight factors for action-angle kick minimization

\begin{tabular}{|c|c|c|c|c|}
\hline Order & $C_{x}$ & $C_{y}$ & $\begin{array}{c}k_{n} l \\
/ k_{n s} l\end{array}$ & $(-1)^{n+1}$ \\
\hline$b_{2}$ & $\beta_{x}^{3 / 2}$ & $\beta_{x}^{1 / 2} \beta_{y}$ & $k_{2} l$ & -1 \\
\hline$b_{3}$ & $\beta_{x}^{2}$ & $\beta_{y}^{2}$ & $k_{3} l$ & 1 \\
\hline$b_{4}$ & $\beta_{x}^{5 / 2}$ & $\beta_{x}^{1 / 2} \beta_{y}^{2}$ & $k_{4} l$ & -1 \\
\hline$b_{5}$ & $\beta_{x}^{3}$ & $\beta_{y}^{3}$ & $k_{5} l$ & 1 \\
\hline$a_{2}$ & $\beta_{x} \beta_{y}^{1 / 2}$ & $\beta_{y}^{3 / 2}$ & $k_{2 s} l$ & -1 \\
\hline$a_{3}$ & $\beta_{x}^{3 / 2} \beta_{y}^{1 / 2}$ & $\beta_{x}^{1 / 2} \beta_{y}^{3 / 2}$ & $k_{3 s} l$ & 1 \\
\hline$a_{4}$ & $\beta_{x}^{2} \beta_{y}^{1 / 2}$ & $\beta_{y}^{5 / 2}$ & $k_{4 s} l$ & -1 \\
\hline$a_{5}$ & $\beta_{x}^{5 / 2} \beta_{y}^{1 / 2}$ & $\beta_{x}^{1 / 2} \beta_{y}^{3 / 2}$ & $k_{5 s} l$ & 1 \\
\hline
\end{tabular}

\section{IR BUMP CORRECTION}

The $\mathbb{R}$ bump correction method $[2,3,4]$ is an elegant way for the operational IR nonlinear Correctionsin a real machine. It creates a local horizontal or vertical orbit bump across the interaction region. The small tune shifts due to the bump are measured with a high resolution phase lock loop ( PLL ) tune measurement system. Since the relations between the tune shifts and the bump amplitudes are different for different orders of nonlinear errors, the IR bump correction is performed order by order by minimizing the polynomial filting coefficientsof the tune shifts.

For example, to correct the sextupole errors in the IR, we minimize the linear term of the tune shifts from the horizontal IR bump with respect to the bump amplitude. According to Hamiltonian perturbation theory, the tune shifts from the sextupoles with the horizontal beam orbit offsets are given by

$$
\left\{\begin{array}{l}
\Delta Q_{x}=\frac{1}{2 \pi} \sum\left(6 b_{2} x_{c o} \beta_{x}\right) d s \\
\Delta Q_{y}=\frac{1}{3 \pi} \sum\left(-6 b_{2} x_{c o} \beta_{y}\right) d s
\end{array} .\right.
$$

Considering the phase advance $\pi$ between the interaction region, we assume that the local horizontal orbit bump is proportional to the $\beta_{x}^{\mathrm{I} / 2}$,

$$
\left\{\begin{array}{cccc}
x_{c o} & \propto & \beta_{x}^{1 / 2}, \text { in one side of IR } \\
x_{c o} & \propto & -\beta_{x}^{1 / 2}, \text { in another side of IR }
\end{array} .\right.
$$

For simplicity, in the following analysis and simulation, we assume that the orbit at one point in the IR bump is proportional to the bump steps. The orbit bump at differentbump steps are given by

$$
x_{c o}=x_{c o, 1} \times k,
$$

$x_{c o, 1}$ is the orbit bump amplitude increment, $k=$ $-N,-(N-1), \ldots,(N-1), N . N$ is the maximum bump 


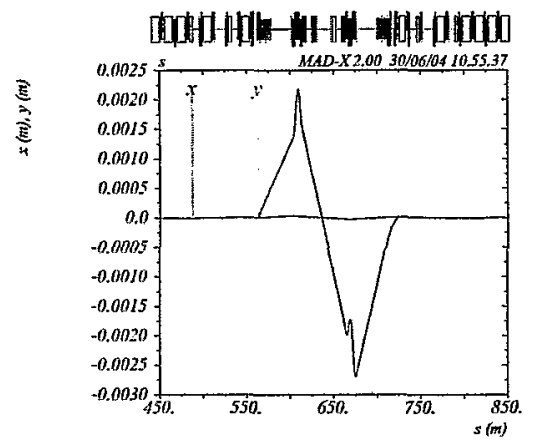

Figure 1: The orbits with the horizontal bump in the RHIC Blue ring IR8.

step No.. Then, the total tune shifts due to the $x$ plane $\mathbb{R}$. bump are

$\left\{\begin{array}{ccc}\Delta Q_{x} & \propto & \left(\sum_{L} b_{2} d s \beta_{x}^{3 / 2}-\sum_{R} b_{2} d s \beta_{x}^{3 / 2}\right) \cdot k \\ \Delta Q_{y} & \propto\left(\sum_{L} b_{2} d s \beta_{x}^{1 / 2} \beta_{y}-\sum_{R} b_{2} d s \beta_{x}^{1 / 2} \beta_{y}\right) \cdot k\end{array}\right.$

. If we use the sextupolecorrectorsin the $\mathbb{R}$ bump to minimize the linearpolynomial term of the tune shifts with the bump steps $k$, we obtain

$$
\left\{\begin{array}{ccc}
\sum_{r} b_{2} d s \beta_{x}^{3 / 2}-\sum_{R} b_{2} d s \beta_{x}^{3 / 2} & = & 0 \\
\sum_{L} b_{2} d s \beta_{x}^{1 / 2} \beta_{y}-\sum_{R} b_{2} d s \beta_{x}^{1 / 2} \beta_{y} & = & 0
\end{array} .\right.
$$

Eq. (8) gives the same weight factors $\beta_{x}^{3 / 2}$ and $\beta_{x}^{1 / 2} \beta_{y}$ as that from the action-angle kick minimization method shown in Table 1.

\section{OFF-LINE COMPARISON}

\section{IR bump Simulation}

The MADX code [5] is used to simulate the IR bump correction. At each bump step, we set the right dipole kicker strengths to produce the desired orbit bump amplitude. MADX calculates the tune shifts at each bump step. By fitting the tune shift with the bump steps, the different orders of polynomial coefficientsare obtained.

For simplicity, in the following we only simulate the IR bump correction in the 8 o'clock interaction region in the RHIC Blue ring. In the simulation, the maximum orbit amplitude in the IR bump is chosen below $\mathbf{5} \mathbf{~ m m}$. Figure 1 shows an example of the orbit with the horizontal IR bump. The orbit leakage due to the IR bump is seen in Figure 1. However, the bump closure is still acceptable. Fig. 2 gives the tune shifts from the simulation. The tune shifts are obtained with respect to that at zero bump amplitude. Up to seven order polynomial fittings are performed, the fitting curve is also shown in Fig. 2. Fig.3 shows the tune shift contributions from the first three polynomial terms.

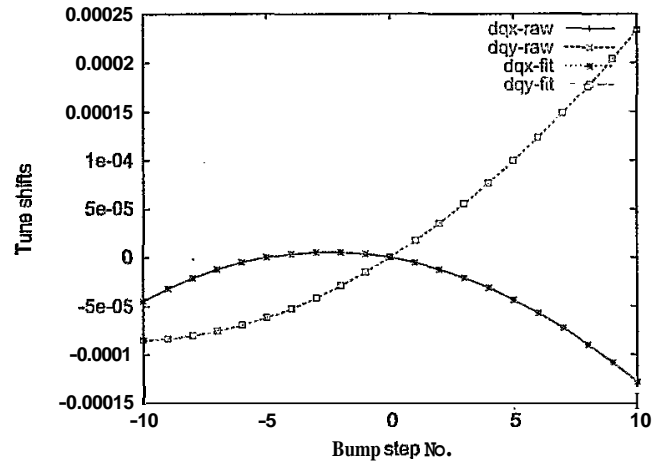

Figure 2: The tune shifts and the polynomial fitting.

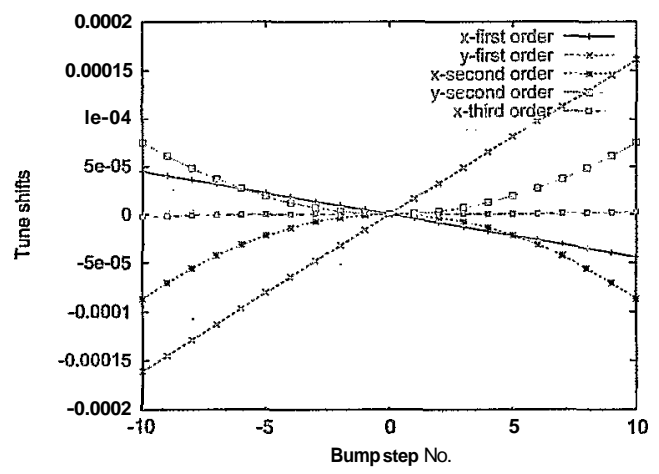

Figure 3: The tune shift contributions from the first three polynomial terms.

\section{Sextupole Correction}

For the horizontal IR bump, the linear terms of the tune shifts are contributedfrom the sextupoles. We use two sextupole correctors bo 7-sx3 and bi8-sx3 to minimize these linear term tune shifts in the Blue IR8. The three dipole kickers bo 7-th4, bi8-th 3 and bi8-th 5 bump produce the desired IR bumps.

Table 2 gives the linear orders from the off-line IR bump simulations. The second block gives the residual linear term of the tune shifts from the IR bump in the IR. The third block gives the two correctors' contributions to the linear terms with $k_{2} l=0.001$. Based on Table 2, the correction strengths for bo $7-\mathrm{s} \times 3$ and bi8-sx3 are calculated to cancel the residual linear terms.

From $\mathbb{R}$ bump simulation, the correction strengths for bo7-sx3 and bi8-sx3 are $-4.54 \times 10^{-3} \mathrm{~m}^{-2}$ and $2.74 \times$ $10^{-3} \mathrm{~m}^{-2}$, respectively. While from the action-anglekick minimization analytical calculation, based on the nonlinear optics model and Eq.(1), the integrated correction strengths for bo 7 -sx 3 and bi 8 -sx3 are $-3.99 \times 10^{-3} \mathrm{~m}^{-2}$ and $2.97^{-3}$ $\mathrm{m}^{-2}$, respectively. There is about a $10 \%$ difference in the correction strength of bo 7 -sx 3 . 


\begin{tabular}{|c|c|c|}
\hline Conditions & Plane & $\begin{array}{c}\text { Linearterm } \\
\text { Coefficient }\end{array}$ \\
\hline \hline only $b_{2}$ & $\mathrm{x}$ & $10.0810^{-5}$ \\
\cline { 2 - 3 } errors & $\mathrm{y}$ & $-9.26 \times 10^{-5}$ \\
\hline \hline bo7-sx3 & $\mathrm{x}$ & $5.65 \times 10^{-6}$ \\
\cline { 2 - 3 }$k_{2} l=0.001 \mathrm{~m}^{-2}$ & $\mathrm{y}$ & $-1.43 \times 10^{-5}$ \\
\hline bi8-sx3 & $\mathrm{x}$ & $-2.74 \times 10^{-5}$ \\
$k_{2} l=0.001 \mathrm{~m}^{-2}$ & $\mathrm{y}$ & $1.01 \times 10^{-5}$ \\
\hline
\end{tabular}

\section{Octupole Correction}

We use the two octupole correctors bo7-oct2 and bi8oct2 to minimize the quadraticterms of the tune shiftsfrom the horizontal IR bump in Blue IR8. The three dipole kickers bo 7-th4, bi8-th3 and B18-TH5 bump produce the desired IR bumps. Table 3 gives the residual quadratic terms from the octupole errors from the IR bump simulations. The correctors bo7-oct2 and bi8-oct2's contributionsto the quadratic terms with $k_{3} l=0.001$ are given, too.

Table 3: IR bump simulation for octupole correction in Blue IR8.

\begin{tabular}{|c|c|c|}
\hline Conditions & Plane & $\begin{array}{c}\text { Quadratic Term } \\
\text { coefficient }\end{array}$ \\
\hline \hline only $\begin{array}{c}\text { errors } \\
\text { erros }\end{array}$ & & $-1.68 \times 10^{-7}$ \\
\cline { 2 - 3 } & $\mathrm{y}$ & $6.40 \times 10^{-8}$ \\
\hline \hline bo7-oct 2 \\
$k_{3} l=0.001 \mathrm{~m}^{-3}$ & $\mathrm{x}$ & $1.58 \times 10^{-7}$ \\
\hline \multirow{2}{*}{$k_{3} l=0.001 \mathrm{~m}^{-3}$} & $\mathrm{y}$ & $-8.74 \times 10^{-8}$ \\
\cline { 2 - 3 } & $\mathrm{x}$ & $7.94 \times 10^{-8}$ \\
\hline
\end{tabular}

From the IR bump correction simulation, the integrated correction strengths for bo7-oct2 and bi8-oct2 are $0.121 \mathrm{~m}^{-3}$ and $-0.029 \mathrm{~m}^{-3}$, respectively. While from the action-angle kick minimization analytical calculation, based on the $\mathbb{R}$ nonlinear modeling, the integtated correction strengths for $\mathrm{b0} 7 \mathrm{~s} \times 3$ and bi8-sx3 are $0.0768 \mathrm{~m}^{-3}$ and $-0.023 \mathrm{~m}^{-3}$,respectively. There are about $30 \%$ difference in the correction strengths of bo7-oct2.

\section{ANALYSIS}

From the off-line IR bump correction and the action angle minimization analytical calculation, we found that there are about $10 \%$ difference for the sextupole corrector strengths, and about $30 \%$ difference for the octupole corrector strengths. They verified the discripancies found in the sextupole and octupole correction strengths from the operational IR bump corrections and the off-line actionangle kicker minimization analytical calculations.

Here we check the ratios of the linear and quadratic terms of two individual sextupole's and octupoles, respec- tively. For sextupoles, according to Eq. (8), the horizontal linear term should be proportional to $\beta_{x}^{3 / 2}$ for two sextupoles if they have the same integrated strength. From IR bump simulations, the ratio of the linear term of the horizontal tune shifts from two sextupolesbo 7 -sx3 and bi8-sx3 with the same integrated strength $0.01 \mathrm{~m}^{-2}$ is

$$
\begin{aligned}
& \left.\Delta \nu_{x}\right|_{b o 7-s x 3}:\left.\Delta \nu_{x}\right|_{b i 8-s x 3} \\
& =5.29 \times 10^{-5}: 27.79 \times 10^{-5} \\
& =1: 5.25
\end{aligned}
$$

The ratio of the $\beta^{3 / 2}$ of the two sextupolesare:

$$
\begin{aligned}
& \beta_{3} /\left.2\right|_{b o 7-s x 3}:\left.\beta_{x}^{3 / 2}\right|_{b i 8-s x 3} \\
& =479.03^{3 / 2}: 1297.89^{3 / 2} \\
& =1: 4.46
\end{aligned}
$$

Therefore, from the IR bump simulation, the ratio of the he ar tune shift terms is not proportional to the ratio of $\beta^{3 / 2}$ for two sextupoles with the same integrated strength. This only reason for the inequality is

$$
\begin{aligned}
& \left.x_{c o}\right|_{b o 7-s x 3}:\left.x_{c o}\right|_{b i 8-s x 3} \\
& \neq\left.\beta_{x}^{1 / 2}\right|_{b o 7-s x 3}:\left.\beta_{x}^{1 / 2}\right|_{b i 8-s x 3} .
\end{aligned}
$$

This guess is verified by the following orbitbump check at these two sextupole correctors. From the TR bump simulation with MADX,

$$
\begin{gathered}
\left.x_{c o}\right|_{b o 7-s x 3}:\left.x_{c o}\right|_{b i 8-s x 3} \\
=1: 1.955, \\
\begin{array}{c}
\left.\beta_{x}^{1 / 2}\right|_{b o 7-s x 3}:\left.\beta_{x}^{1 / 2}\right|_{b i 8-s x 3} \\
=1: 1.646 .
\end{array}
\end{gathered}
$$

If we substitute the orbit ratio of $x_{c o}$ instead of the ratio of $\beta_{x}^{1 / 2}$ into Eq. (4), we get the horizontal tune shift contribution ratio from the $t w o$ sextupoles:

$$
\begin{gathered}
\left.\left(x_{c o} \beta_{x}\right)\right|_{b o 7-s x 3}:\left.\left(x_{c o} \beta_{x}\right)\right|_{b i 8-s x 3} \\
=1: 5.30
\end{gathered}
$$

which is much closer to the linear term tune shift ratio we get from the $\mathbb{R}$ bump simulation.

Similarly, we check the quadratic term tune shift ratios of two individual octupolesbo7-oct2 and bi8-oct2 with the same integrated strength $0.001 \mathrm{~m}^{-3}$. From the $\mathbb{R}$ bump simulation, we get

$$
\begin{aligned}
& \left.\Delta \nu_{x}\right|_{\text {bo7-oct } 2}:\left.\Delta \nu_{x}\right|_{\text {bis-oct } 2} \\
& =1.58 \times 10^{-7}: 7.94 \times 10^{-8} \\
& =1.996: 1
\end{aligned}
$$

However,

$$
\begin{aligned}
& \left.\beta_{x}^{2}\right|_{b o 7-s x 3}:\left.\beta_{x}^{2}\right|_{b i 8-s x 3} \\
& =1042.11^{\prime}: 577.37^{\prime} \\
& =3.261: 1
\end{aligned}
$$

The ratio of the tune shifts are not equal to the ratio of $\beta_{x}^{2}$ as assumed from Table 1 , either. The ratio of orbit amplitudes at the two octupolesis:

$$
\begin{aligned}
& \left.x_{c o}\right|_{b o 7-o c t 2}:\left.x_{c o}\right|_{b i 8-o c t 2} \\
& =1.051: 1
\end{aligned}
$$


Substituting $x_{c o}$ ratio instead of $\beta_{x}^{1 / 2}$ ratio to calculate the tune shifts due to octupoles, we obtain the horizontal tune shift contributionratio \&om the two octupoles:

$$
\begin{gathered}
\left.\left(x_{c o}^{2} \beta_{x}\right)\right|_{b o 7-s x 3}:\left.\left(x_{c o}^{2} \beta_{x}\right)\right|_{b i 8-s x 3} \\
=1.995: 1
\end{gathered}
$$

which is almost the same as that from the IR bump simulation.

\section{DISCUSSION}

From the abovecalculation, we find the difference of the correction strengths from the IR bump correction and the action-angle kick minimization comes from the fact that the horizontal orbit is not exactly proportional to the $\beta_{x}^{1 / 2}$. The source for this differenceis that the phase advance over the interaction region is not exactly equal to $\pi$ and there is a small phase advance in the triplet.

Presenting the phase advance as $\Delta \Psi=\pi+\mathrm{AI}$ ) in another IR side, one can get the ratio of orbit positions on the left and right sides of the interaction region as

$$
\begin{aligned}
\frac{x_{c o, L}}{x_{c o, R}} & =\frac{\sqrt{\beta_{x, L}} \sin \left(\Psi_{0}\right)}{\frac{\sqrt{\beta_{x, R}} \sin \left(\Psi_{0}+\Delta \Psi\right)}{\sqrt{\beta_{x, L}}}\left(1-\cot \left(\Psi_{0}\right) \Delta \psi\right) .} \\
& \simeq-\frac{\sqrt{\beta_{x, R}}}{\sqrt{x_{2}}}
\end{aligned}
$$

Although A $\$$ is small, the cot $\psi_{0}$ can reach 15 units for RHIC IR bump. It leads to considerabledifferencebetween the ratio of the orbits and the ratio of $\sqrt{\beta}_{x}$. Then the correction strengths from the IR bump correction are not the same as that from the action-anglekick minimization analytical prediction.

Action-anglekick minimization ignores the small phase change in the IR bump on both sides of the IP, while the IR bump correction method takes into account the phase shifts. Base on the Hamiltonian perturbation theory, sextupoles could introduce $Q_{x}=p, 3 Q_{x}=p, Q_{x} \pm 2 Q_{y}=p$ resonances. To fully correct all the resonances, we should minimize all the followingresonancestrengths [6], that is,

$$
\left\{\begin{array}{ccc}
\sum_{j} k_{2} l \beta_{x}^{1 / 2} \beta_{y} e^{i \Psi_{x}} & \longrightarrow & 0 \\
\sum_{j} k_{2} l \beta_{x}^{3 / 2} e^{i \Psi_{x}} & \longrightarrow & 0 \\
\sum_{j} k_{2} l \beta_{x}^{3 / 2} e^{i 3 \Psi_{x}} & \longrightarrow & 0 \\
\sum_{j} k_{2} l \beta_{x}^{1 / 2} \beta_{y} e^{i\left(\Psi_{x}-2 \Psi_{y}\right)} & \longrightarrow & 0 \\
\sum_{j} k_{2} l \beta_{x}^{1 / 2} \beta_{y} e^{i\left(\Psi_{x}+2 \Psi_{y}\right)} & \longrightarrow & 0
\end{array}\right.
$$

Octupolesinduce $4 Q_{x}=p, 4 Q_{y}=p, 2 Q_{x}=p, 2 Q_{y}=p$, $2 Q_{x} \pm 2 Q_{y}=p$ resonances. To correct all the resonances, we should minimize all the followingresonance strengths, that is,

$$
\begin{array}{ccc}
\sum_{j} k_{3} l \beta_{x}^{2} e^{i 4 \Psi_{x}} & \longrightarrow & 0 \\
\sum_{j} k_{3} l \beta_{y}^{2} e^{i 4 \Psi_{y}} & \longrightarrow & 0 \\
\sum_{j} k_{3} l \beta_{x} \beta_{y} e^{i 2 \Psi_{x}} & \longrightarrow & 0 \\
\sum_{j} k_{3} l \beta_{i x}^{2} e^{i 2 \Psi_{x}} & \longrightarrow & 0 \\
\sum_{j} k_{3} l \beta_{x} \beta_{y} e^{i 2 \Psi_{y}} & \longrightarrow & 0 \\
\sum_{j} k_{3} l \beta_{y}^{2} e^{i 2 \Psi_{y}} & \longrightarrow & 0 \\
\sum_{j} k_{3} l \beta_{x} \beta_{y} e^{i\left(2 \Psi_{x}+2 \Psi_{y}\right)} & \longrightarrow & 0 \\
\sum_{j} k_{3} l \beta_{x} \beta_{y} e^{i\left(2 \Psi_{x}-2 \Psi_{y}\right)} & \longrightarrow & 0
\end{array}
$$

So the two methods, IR bump correction and the actionangle kick minimization, have different approximationsin the betatron phase advance. Action-anglekicker minimization ignores the not exact $\pi$ phase jump across the $\mathbb{P}$ and the samll phase change in the one side triplet. The IR bump correction method uses a local orbit bump to minimize the introduced polynomial terms of tune shifts. However, the tune shifts from IR bump are proportional to $\sin ^{n} \Phi$, instead of $e^{i n \Phi}$ from Eqs. (19) - (20).

The action-anglekick minimization is used for $\boldsymbol{R}$ nonlinear field correction off line up to $b_{10}$ at CERN [7]. The IR bump correction is applicable for the on linear IR nonlinear field correction. It has been verified and used in the RHIC IR nonlinear corrections. Limited by the bump amplitude and the tune measurementresolution, it is generally used for the lower order nonlinear field error corrections.

\section{CONCLUSION}

The correction strengths from the off-line IR bump correction simulation are compared to that from the actionangle kick minimization. It verifies that there are some discrepancies in the correctionstrengthsfrom these two methods. This is caused by the fact that the not exact $\pi$ phase advance between the two sides of the interaction region, which makes that the bump orbit not exactlyproportionally to the $\beta^{1 / 2}$. Both methods make different approximations in the betatron phase advances. The action-anglekick minimization is used for IR nonlinear field correction off line, while the IR bump correction is applicable for the on-line IR nonlinear field correction.

\section{REFERENCES}

[1] J. Wei, Proceedings of the Workshop on LHC Interaction Region Correction Systems, BNL, May 6-7,1999.

[2] J-P. Koutchouk, F. Pilat, V. Ptitsyn, et al, PAC2001.

[3] F. Pilat, V. Ptitsyn, et al., EPAC2002, Paris, France.

[4] Y. Luo, F. Pilat, V. Ptitsyn, et al., BNL. C-AD AP Note 160, Aug. 2004.

[5] Madx code, CERN, http://madhome.cem.ch/mad/.

[6] H. Wiedemann, Particle Accelerator Physics: Nonlinear and Higher-orderBeam Dynamics, Springer Press, 1994.

[7] H. Grote, et al, LHC-project-note 197. 